\title{
Fuzziness in analytic network process under interval numbers for criteria and alternatives
}

\begin{abstract}
Because of the lack of data or knowledge or limited time, decision makers could not express their experiences exactly, perhaps they prefer interval numbers for such situations. Whenever uncertainty is involved in the decision making process, fuzzy and stochastic models would be arisen. Recently, fuzzy theory for Multiple Attribute Decision Making (MADM) under interval numbers has attracted a lot of researchers. This paper deals with a fuzzy MADM approach under interval numbers. We propose to apply the approach for Analytic Network Process (ANP) as a new class of decision making methods. The interval numbers are formed for criteria weights and values that have effect on alternativesôvalues. The process of this method is clarified by an example.
\end{abstract}

Keyword: Analytic network process; Fuzzy set; Intervals numbers 